\title{
Reduction of energy chirp by the wake of coherent synchrotron radiation
}

\author{
R. A. Bosch \\ Synchrotron Radiation Center, University of Wisconsin-Madison, 3731 Schneider Drive, Stoughton, Wisconsin 53589, USA
}

(Received 15 April 2010; published 19 November 2010)

\begin{abstract}
When an energy-chirped bunch is undercompressed in a magnetic chicane, the compressed bunch also has an energy chirp. The chirp may be decreased by sending the bunch through bending magnets, since the largest energy loss from the wake of coherent synchrotron radiation occurs in the tail of a typical bunch. We obtain formulas for the dechirping of a rectangular bunch by short magnets, and apply this dechirping technique in a design for a free-electron laser.
\end{abstract}

DOI: 10.1103/PhysRevSTAB.13.110702

PACS numbers: 41.60.Cr, 41.60.Ap, 29.27.Bd

\section{INTRODUCTION}

An ultrarelativistic bunch of electrons with an energy chirp may be compressed by sending it through a magnetic chicane. If the bunch is undercompressed, so that its head and tail are not exchanged, the compressed bunch also has an energy chirp with increasing energy towards the tail. In a seeded free-electron laser (FEL), the performance may be improved by removing the chirp, i.e., dechirping the bunch [1].

If the bunch is accelerated after compression, dechirping may be accomplished by off-crest acceleration in the linac. However, off-crest acceleration may incur a significant expense for additional radiofrequency (rf) cavities. When a typical bunch passes through a bending magnet, the largest energy loss from the wake of coherent synchrotron radiation (CSR) occurs in the bunch's tail [2-4]. This suggests that it may be cost effective to dechirp a bunch with the CSR wake of bending magnets.

To minimize transverse emittance growth from CSRinduced energy loss in dispersive regions, we consider bending magnets with small deflection angles-much smaller than those in the bunch compressor-arranged so that position and momentum dispersion are much smaller than in the bunch compressor. For a small deflection angle, the CSR is approximated by a short magnet, whose length is much smaller than the formation length of the bunch's steady-state CSR. We obtain formulas for the dechirping of a rectangular bunch, whose constant current profile is ideal for a seeded FEL [1]. The formulas may be used to estimate the number of bending magnets needed to dechirp a given bunch, and the required distance between them.

In a typical seeded-FEL design, a chirped bunch with charge of several hundred $\mathrm{pC}$ and normalized transverse emittance of $\sim 1 \mu \mathrm{m}$ rad is compressed at a beam energy of several hundred $\mathrm{MeV}$ to obtain a current of $\sim 1 \mathrm{kA}$ [1,5-11]. As an example, we study a single-stage compression design for the Wisconsin FEL (WiFEL) [12]. According to analytic modeling and tracking simulations, $\sim 15$ short bending magnets provide sufficient dechirping so that on-crest acceleration may be used to reduce $\mathrm{rf}$ expense. Because the number of bending magnets is not large, dechirping with CSR may also be feasible in similar FEL designs.

\section{THE WISCONSIN FEL}

Figure 1 illustrates a design for WiFEL, in which a 200-pC bunch with 3-keV Gaussian energy spread and normalized transverse emittance of $1 \mu \mathrm{m}$ rad is accelerated in TESLA 1.3-GHz and 3.9-GHz superconducting rf cavities. The bunch is compressed at beam energy of $400 \mathrm{MeV}$ in a three-magnet chicane with deflection angles of $5^{\circ},-10^{\circ}$, and $5^{\circ}$. The compressed bunch is accelerated to $1.7 \mathrm{GeV}$ in $1.3-\mathrm{GHz}$ superconducting rf cavities and distributed to an FEL by an isochronous beam spreader that incorporates a three-stage if separator tree [11]. To provide a dechirped bunch for the FEL, the compressed bunch is accelerated at a linac phase of $40^{\circ}$ after crest.

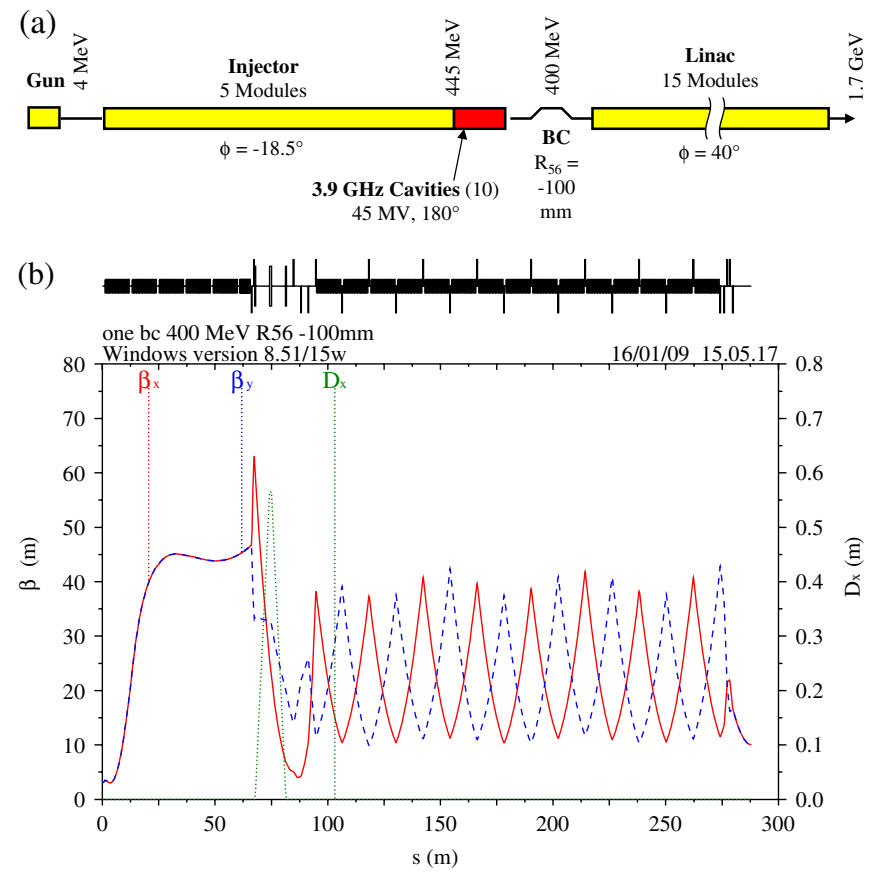

FIG. 1. Single-stage bunch compressor for WiFEL. (a) Schematic diagram. (b) Lattice functions. 
We simulate the bunch by tracking with the ELEGANT code [13], including the CSR wake in magnets and drift regions (computed with the method of Ref. [3]), the linac's geometric wake, the resistive-wall wake of the beam spreader vacuum chambers, and incoherent synchrotron radiation. To study macroscopic bunch properties, we track without the wake of longitudinal space charge (LSC), reducing numerical noise by using Halton sequences [14] to populate the initial phase space with 4 million particles.

For a tracking simulation without LSC, Fig. 2(a) shows the longitudinal phase space immediately after compression. The bunch has an approximately linear chirp of $2.3 \times 10^{19} \mathrm{~V} / \mathrm{s}$. After acceleration to $1.7 \mathrm{GeV}$ at a linac phase of $40^{\circ}$ after crest, Fig. 2(b) shows that the bunch chirp is reduced by $1.4 \times 10^{19} \mathrm{~V} / \mathrm{s}$. Comparing on-crest and off-crest acceleration indicates that accelerating off crest reduces the chirp by $9 \times 10^{18} \mathrm{~V} / \mathrm{s}$, while the geometric wake of the rf cavities reduces the chirp by $5 \times 10^{18} \mathrm{~V} / \mathrm{s}$. The remaining chirp of $9 \times 10^{18} \mathrm{~V} / \mathrm{s}$ is eliminated by the CSR wake of the beam spreader, as shown in Fig. 2(c).

Throughout the compressed bunch evolution, the longitudinal distribution is nearly frozen. Figure 2(d) shows the current profile at the spreader exit. Except for current spikes at the head and tail, the profile is approximately a rectangular distribution with current of $1 \mathrm{kA}$ and duration of 200 fs. If the compressed bunch can be dechirped $9 \times 10^{18} \mathrm{~V} / \mathrm{s}$ by CSR wakes, the expense for rf cavities may be reduced by accelerating on crest.
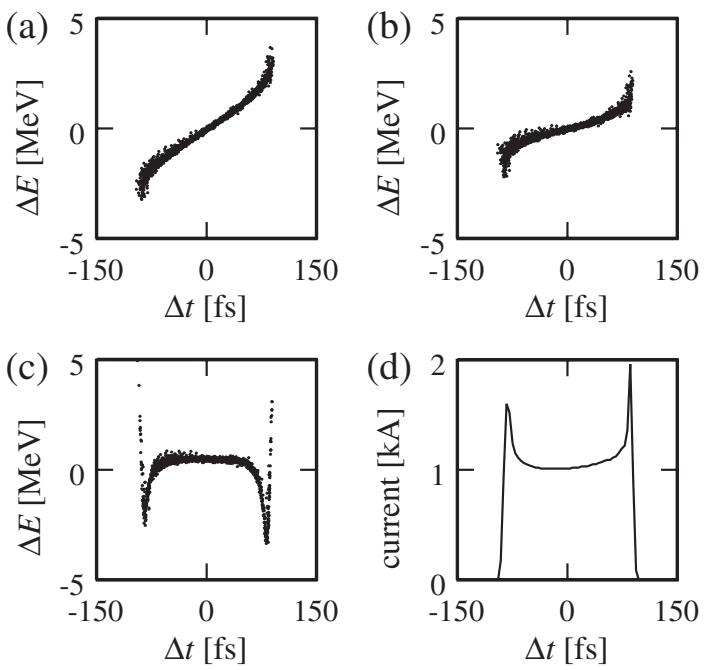

FIG. 2. Evolution of longitudinal phase space after bunch compression. The bunch tail is on the right. (a) Chirped bunch at the exit of the bunch compressor, at beam energy of $400 \mathrm{MeV}$. (b) Bunch with reduced chirp at the linac exit where the beam energy is $1.7 \mathrm{GeV}$. (c) Dechirped bunch at the exit of the beam spreader at beam energy of $1.7 \mathrm{GeV}$. (d) Current distribution of the longitudinally frozen bunch, evaluated at the exit of the beam spreader.

\section{DECHIRPING A RECTANGULAR BUNCH}

For an ultrarelativistic bunch with a rectangular current profile, consider the CSR wake of an infinitesimally short magnet with deflection angle $\theta$ that obeys $1 / \gamma \ll \theta \ll$ $\pi / 2$, where $\gamma$ is the bunch's relativistic factor [4]. The wake is the sum of the wake of a bunch that is suddenly stopped at the magnet entrance (the "entrance edge" wake) with the wake of a bunch that is suddenly accelerated in a different direction at the magnet exit (the "exit edge" wake). In the convention where a negative wake describes energy loss in $\mathrm{V} / \mathrm{m}$, the longitudinal wake at distance $z$ downstream of the magnet, experienced by an on-axis electron that exits the magnet at time $\Delta t$, is $[4,15]$

$$
\begin{aligned}
W(z, \Delta t)= & \frac{-N q}{\pi \varepsilon_{0} r_{b}^{2}}\left[\int_{\Delta t-\left[\left(\sqrt{z^{2}+r_{b}^{2}}-z\right) / c\right]}^{\Delta t} f(t) d t-(\cos \theta)^{-1}\right. \\
& \left.\times \int_{\Delta t-\left[\left(\sqrt{z^{2}+r_{b}^{2}} \cos \theta-z\right) / c\right]}^{\Delta t-[(z \cos \theta-z) / c]} f(t) d t\right] .
\end{aligned}
$$

Here, $N$ is the number of electrons in the bunch, $q>0$ is the magnitude of the electron charge, $\varepsilon_{0}$ is the permittivity of free space, $r_{b}$ is the bunch radius, $f(\Delta t)$ is the bunch's normalized longitudinal profile, and $c$ is the speed of light. For a rectangular bunch of duration $t_{b}$ with $\Delta t=0$ at its center, the profile is $f(\Delta t)=H\left(t_{b} / 2-|\Delta t|\right) / t_{b}$, where $H$ is the Heaviside function. Equation (1) becomes

$$
\begin{aligned}
W(z, \Delta t)= & \frac{-N q}{\pi \varepsilon_{0} r_{b}^{2} t_{b}}\left(\max \left[\frac{-t_{b}}{2}, \min \left(\Delta t, \frac{t_{b}}{2}\right)\right]\right. \\
& -\max \left[\frac{-t_{b}}{2}, \min \left(\Delta t-\frac{\sqrt{z^{2}+r_{b}^{2}}-z}{c}, \frac{t_{b}}{2}\right)\right] \\
& -(\cos \theta)^{-1}\left\{\max \left[\frac{-t_{b}}{2}, \min \left(\Delta t-\frac{z \cos \theta-z}{c}, \frac{t_{b}}{2}\right)\right]\right. \\
& \left.\left.-\max \left[\frac{-t_{b}}{2}, \min \left(\Delta t-\frac{\sqrt{z^{2}+r_{b}^{2}} \cos \theta-z}{c}, \frac{t_{b}}{2}\right)\right]\right\}\right) .
\end{aligned}
$$

Figure 3 illustrates the wake given by Eq. (2) for a 0.012$\operatorname{rad}\left(0.69^{\circ}\right)$ deflection of a $200-\mathrm{pC}$ bunch with duration of $200 \mathrm{fs}$ and radius of $40 \mu \mathrm{m}$. The positive entrance-edge wake gradually moves ahead of the bunch, the negative exit-edge wake moves with the bunch, and the total wake is given by their sum. The integrated wake gives the accumulated energy change over the interval $(0, z)$. As the bunch propagates, the entrance-edge wake overtakes the bunch and the integrated wake develops a nonlinear chirp.

Shortly downstream of the magnet, Fig. 3(a) shows that the entrance-edge wake is slightly ahead of the exit-edge wake. Their incomplete cancellation causes a large total wake at the bunch tail and slightly ahead of the bunch. In Fig. 3(b), the entrance-edge wake completely overtakes the bunch where $z=z_{c} \equiv c t_{b} /(1-\cos \theta)$. Figure 3(c) shows that further propagation of the rectangular bunch does not 

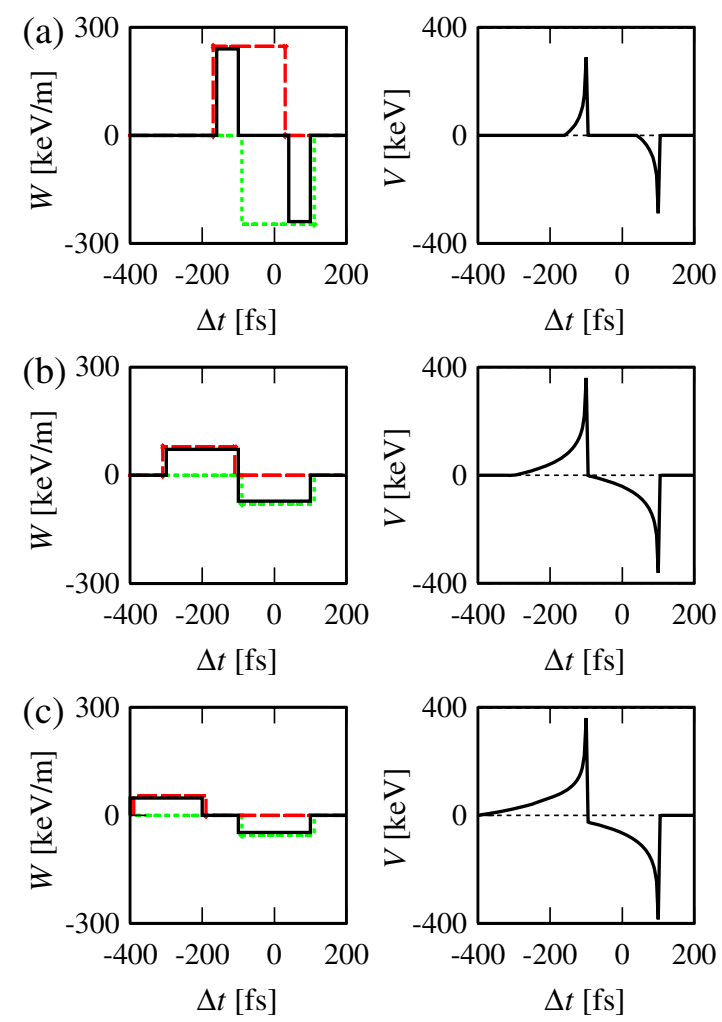

FIG. 3. Longitudinal wakes downstream of a 0.012-rad deflection of a rectangular bunch with charge of $200 \mathrm{pC}$, duration of $200 \mathrm{fs}$, and radius of $40 \mu \mathrm{m}$. Negative wakes correspond to energy loss. Left-hand plots: a dashed red curve shows the entrance-edge wake, a short-dashed green curve shows the exit-edge wake (which is proportional to the bunch current profile). A solid black curve shows the total wake given by their sum. Right-hand plots: integrated total wake. (a) Wakes at a distance of $0.25 \mathrm{~m}$. (b) At a distance of $0.833 \mathrm{~m}$, the entranceedge wake completely overtakes the bunch. (c) Wakes at a distance of $1.25 \mathrm{~m}$.

change its chirp, because the resistive exit-edge wake causes uniform energy loss. For deflection angles with $1 / \gamma \ll \theta \ll \pi / 2$, the integrated wake at distance $z_{c} \equiv$ $c t_{b} /(1-\cos \theta)$ is nearly independent of the deflection angle $\theta$, differing only at the tip of the bunch tail and immediately ahead of the bunch.

To analyze the wake, we approximate the integrands as constant in Eq. (1) to obtain a small-radius approximation that applies for $z \gg \max \left(r_{b}, r_{b}^{2} / c t_{b}\right)[4]$

$$
\begin{aligned}
W(z, \Delta t) \approx & \frac{-N q\left(\sqrt{z^{2}+r_{b}^{2}}-z\right) Z_{0}}{\pi r_{b}^{2}} \\
& \times\left[f(\Delta t)-f\left(\Delta t+\frac{z(1-\cos \theta)}{c}\right)\right],
\end{aligned}
$$

where $Z_{0}=\left(\varepsilon_{0} c\right)^{-1}=120 \pi$ ohms. Using Eq. (3) to calculate the integrated wake of a rectangular bunch over the interval $(0, z)$ gives

$$
\begin{aligned}
V(z, \Delta t)= & \int_{0}^{z} W\left(z^{\prime}, \Delta t\right) d z^{\prime}=\frac{-N q Z_{0}}{2 \pi t_{b}} H\left(\frac{t_{b}}{2}-|\Delta t|\right) \\
& \times H\left(z-\frac{c\left(t_{b} / 2-\Delta t\right)}{1-\cos \theta}\right)\left[G\left(\frac{z}{r_{b}}\right)\right. \\
& \left.-G\left(\frac{c\left(t_{b} / 2-\Delta t\right)}{r_{b}(1-\cos \theta)}\right)\right]+\frac{N q Z_{0}}{2 \pi t_{b}} H\left(\frac{-t_{b}}{2}-\Delta t\right) \\
& \times\left[G\left(\min \left[\frac{z}{r_{b}}, \frac{c\left(t_{b} / 2-\Delta t\right)}{r_{b}(1-\cos \theta)}\right]\right)\right. \\
& \left.-G\left(\min \left[\frac{z}{r_{b}}, \frac{c\left(-t_{b} / 2-\Delta t\right)}{r_{b}(1-\cos \theta)}\right]\right)\right]
\end{aligned}
$$

where $G(x) \equiv x \sqrt{1+x^{2}}+\ln \left(x+\sqrt{1+x^{2}}\right)-x^{2}$.

In the one-dimensional (1D) ultrarelativistic approximation [3] obtained by the limit $r_{b} \rightarrow 0$, Eq. (3) becomes

$$
W(z, \Delta t) \approx \frac{-N q Z_{0}}{2 \pi z}\left[f(\Delta t)-f\left(\Delta t+\frac{z(1-\cos \theta)}{c}\right)\right] .
$$

Integrating Eq. (5) gives the 1D approximation for the integrated wake of a rectangular bunch:

$$
\begin{aligned}
V(z, \Delta t)= & \frac{-N q Z_{0}}{2 \pi t_{b}} H\left(\frac{t_{b}}{2}-|\Delta t|\right) H\left(z-\frac{c\left(t_{b} / 2-\Delta t\right)}{1-\cos \theta}\right) \\
& \times \ln \left(\frac{z(1-\cos \theta)}{c\left(t_{b} / 2-\Delta t\right)}\right)+\frac{N q Z_{0}}{2 \pi t_{b}} H\left(\frac{-t_{b}}{2}-\Delta t\right) \\
& \times \ln \left(\frac{\min \left[z(1-\cos \theta), c\left(t_{b} / 2-\Delta t\right)\right]}{\min \left[z(1-\cos \theta), c\left(-t_{b} / 2-\Delta t\right)\right]}\right) .
\end{aligned}
$$

In the 1D approximation, the dechirping wake of a rectangular bunch, given by the integrated wake at $z=z_{c}$, is

$$
\begin{aligned}
V\left(z_{c}, \Delta t\right) & =\frac{-N q Z_{0}}{2 \pi t_{b}}\left[H\left(\frac{t_{b}}{2}-|\Delta t|\right) \ln \left(\frac{t_{b}}{t_{b} / 2-\Delta t}\right)\right. \\
& \left.-H\left(\frac{t_{b}}{2}-\left|\Delta t+t_{b}\right|\right) \ln \left(\frac{t_{b}}{-t_{b} / 2-\Delta t}\right)\right] .
\end{aligned}
$$

In Eqs. (6) and (7), the integrated wake approaches $-\infty$ at the tip of the bunch tail and $+\infty$ immediately ahead of the bunch, because of the simultaneous singular assumptions of a rectangular ultrarelativistic bunch, a zero-length magnet, and a zero-radius bunch. Elsewhere, Eq. (7) agrees with the integrated wake computed for a finite-radius bunch.

In the 1D approximation, the integrated wake at distance $z_{c} \equiv c t_{b} /(1-\cos \theta)$ is independent of the deflection angle $\theta$. Away from the tip of the tail, the integrated wake within the bunch has chirp (in units of $\mathrm{V} / \mathrm{s}$ ) given by the derivative of Eq. (7),

$$
\frac{\partial V\left(z_{c}, \Delta t\right)}{\partial \Delta t}=\frac{-N q Z_{0}}{2 \pi t_{b}\left(t_{b} / 2-\Delta t\right)} .
$$

Thus, the bunch is dechirped nonlinearly as it travels downstream of the magnet over the dechirping length $z_{c}$ given by 


$$
z_{c} \equiv c t_{b} /(1-\cos \theta)
$$

The dechirping at the center of the bunch, given by Eq. (8) for $\Delta t=0$, is

$$
\frac{\partial V\left(z_{c}, \Delta t=0\right)}{\partial \Delta t}=\frac{-N q Z_{0}}{\pi t_{b}^{2}} .
$$

For a typical geometry where $z_{c} \gg \max \left(r_{b}, r_{b}^{2} / c t_{b}\right)$, the longitudinal wake is nearly the same for off-axis electrons [4], providing nearly uniform dechirping across the bunch's transverse cross section.

For WiFEL ( $\left.N q=200 \mathrm{pC}, t_{b}=200 \mathrm{fs}\right)$, Eq. (10) gives the value of $-6 \times 10^{17} \mathrm{~V} / \mathrm{s}$. The dechirping that is now provided by off-crest acceleration $\left(-9 \times 10^{18} \mathrm{~V} / \mathrm{s}\right)$ is expected from 15 short bending magnets separated by distances greater than or equal to the dechirping length $z_{c}$. Because the estimated number of bending magnets is not large, dechirping by CSR wakes appears feasible.

\section{CHICANE DECHIRPER}

For WiFEL dechirping, we consider a periodic focusing cell that contains two four-magnet chicanes. Figure 4 displays a design for beam energy of $1.7 \mathrm{GeV}$, in which each bending magnet has a length of $0.2 \mathrm{~m}$ and deflection angle $\theta=0.012 \operatorname{rad}\left(0.69^{\circ}\right)$. The length of each chicane's first (and third) drift region, when added to one-half of the magnet length, nearly equals $z_{c}=0.8333 \mathrm{~m}$. Each chicane has $R_{56}=-2 z_{c} \theta^{2}=-4 c t_{b}=-240 \mu \mathrm{m}$ (in the convention where $R_{56}$ is negative for a chicane).

Figure 5 displays simulations without the LSC wake for a compressed bunch that is accelerated on crest. Figure 5 (a) shows the chirped bunch when no dechirping cells are used. We tracked with one, two, three, or four dechirping cells immediately upstream of the linac, where the beam energy is $400 \mathrm{MeV}$. As expected, the chirp in the center of the bunch is smallest when two dechirping cells

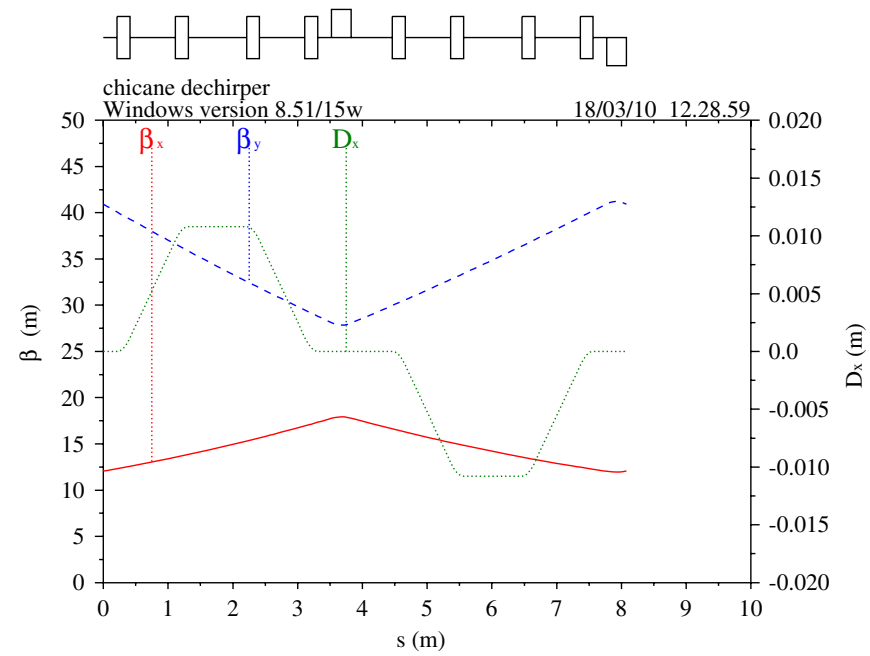

FIG. 4. Lattice functions for a dechirping cell that contains two chicanes.
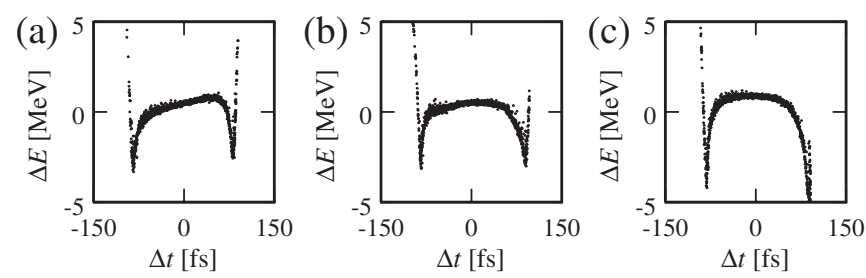

FIG. 5. Longitudinal phase space at the exit of the beam spreader for on-crest acceleration of the compressed bunch. (a) No dechirping cells. (b) Two dechirping cells, which contain a total of 16 bending magnets in four chicanes, at beam energy of $400 \mathrm{MeV}$. (c) Two dechirping cells at beam energy of $1.7 \mathrm{GeV}$.

(containing a total of 16 short magnets) are used, as shown in Fig. 5(b). Figure 5(c) shows that effective dechirping is also provided by two dechirping cells located at the linac exit, where the beam energy is $1.7 \mathrm{GeV}$. The transverse phase space is not degraded by using the dechirping chicanes.

Two dechirping cells have an $R_{56}$ value of $-960 \mu \mathrm{m}$, sufficiently large to increase the microbunching gain of the
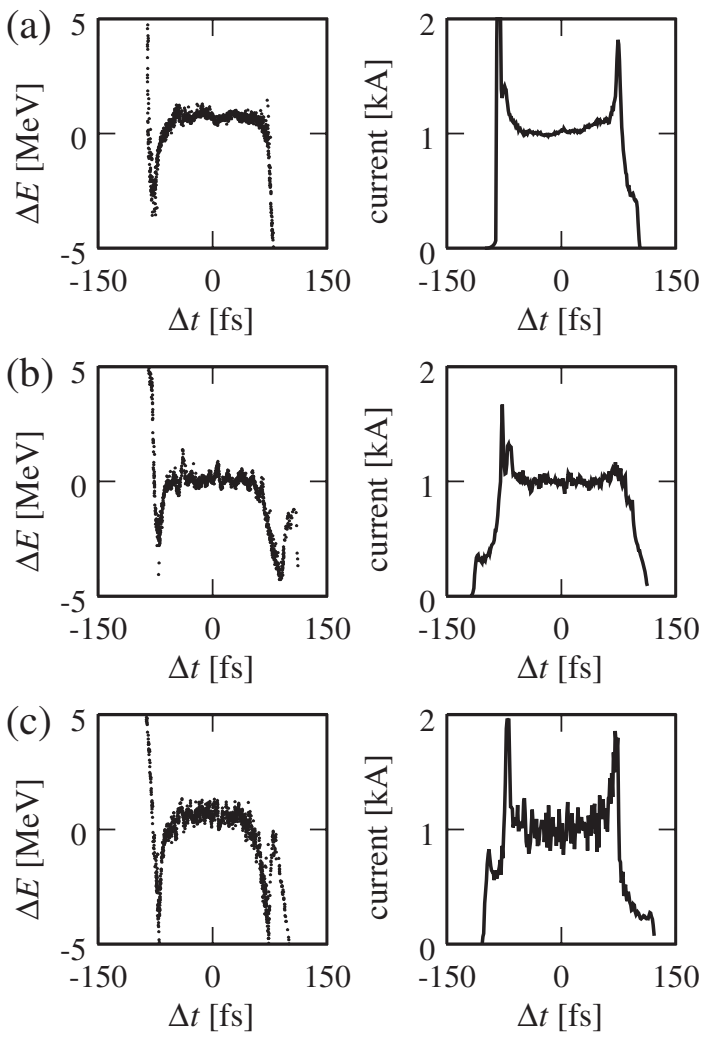

FIG. 6. Simulations of microbunching from shot noise. The left-hand plots show the longitudinal phase space while the righthand plots show 200-bin current histograms at the exit of the beam spreader. (a) Dechirping the compressed bunch by $40^{\circ}$ offcrest acceleration. (b) Dechirping by two dechirping cells at beam energy of $400 \mathrm{MeV}$ (16 bending magnets in four chicanes). (c) Dechirping by two dechirping cells at beam energy of $1.7 \mathrm{GeV}$. 
FEL design $[5,12,16]$. To approximate microbunching from the shot noise of a 200-pC bunch containing $1.25 \times$ $10^{9}$ electrons, we include the LSC wake in the tracking of 8 million randomly distributed particles, starting at the electron gun energy of $4 \mathrm{MeV}$. We reduce the LSC wake upstream of the bunch compressor by the factor $12.5=$ $\left(1.25 \times 10^{9} / 8 \times 10^{6}\right)^{1 / 2}$, so that the energy fluctuations at its entrance represent the bunch's $1.25 \times 10^{9}$ electrons [17]. Figure 6(a) shows the bunch at the FEL when offcrest acceleration of the compressed bunch is used to obtain a dechirped bunch. Figure 6(b) shows a bunch that has passed through two dechirping cells at beam energy of $400 \mathrm{MeV}$, after which it is accelerated to $1.7 \mathrm{GeV}$ on crest and propagated through the beam spreader. Figure 6(c) shows a bunch that has been accelerated on crest from $400 \mathrm{MeV}$ to $1.7 \mathrm{GeV}$, and then propagated through two dechirping cells and the beam spreader. The microbunching is increased when the dechirping cells are used, marginally satisfying the FEL requirements for energy modulations less than $0.5 \mathrm{MeV}$ and relative current modulations less than $10 \%$. A laser heater [18] may improve the FEL performance.

\section{ISOCHRONOUS ARC DECHIRPER}

To reduce the microbunching caused by dechirping magnets, we designed the isochronous $\left(R_{56}=0\right)$ achromatic arc [19] shown in Fig. 7, which contains three short bending magnets whose deflection angles are $0.012 \mathrm{rad}$. Four arcs were concatenated (with quadrupoles and drift regions between them) to construct an isochronous dechirping cell containing 12 bending magnets that produces no net deflection or translation. Figure 8 shows tracking without LSC for on-crest acceleration of the compressed bunch. Effective dechirping is obtained with a dechirping

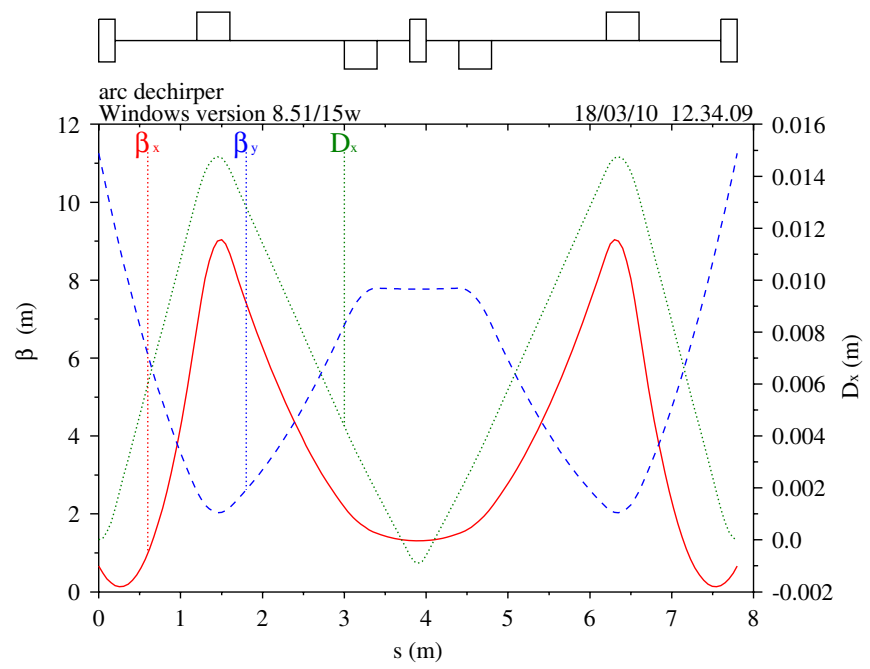

FIG. 7. Lattice functions for an isochronous achromatic dechirping arc that contains three short bending magnets.
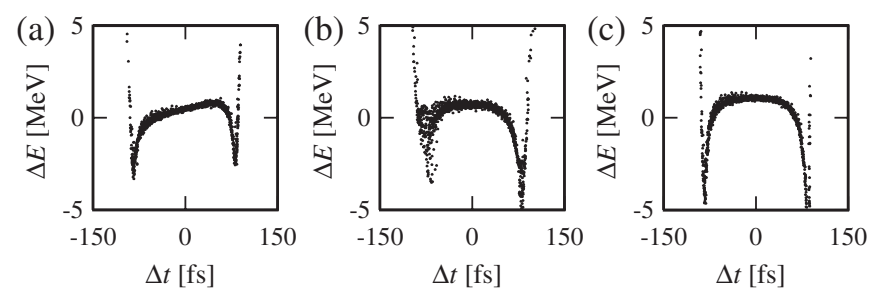

FIG. 8. Longitudinal phase space at the exit of the beam spreader for on-crest acceleration of the compressed bunch. (a) No dechirping arcs. (b) Four isochronous dechirping arcs (containing a total of 12 bending magnets) at beam energy of $400 \mathrm{MeV}$. (c) Four isochronous dechirping arcs at beam energy of $1.7 \mathrm{GeV}$.

cell immediately before the linac at beam energy of $400 \mathrm{MeV}$, or with a dechirping cell after the linac at beam energy of $1.7 \mathrm{GeV}$. The transverse phase space of the bunch's central region (which will be seeded further downstream for the FELs) is not degraded by the dechirping arcs.

In Fig. 9, microbunching from shot noise is approximated by tracking 8 million particles from the electron-gun exit to an FEL, with LSC wakes included, in which the LSC wake upstream of the bunch compressor is reduced by the factor 12.5. With isochronous bending arcs used for dechirping, the microbunching is comparable to that in Fig. 6(a), where dechirping is obtained by off-crest acceleration of the compressed bunch. The microbunching is sufficiently small that a laser heater is not required.
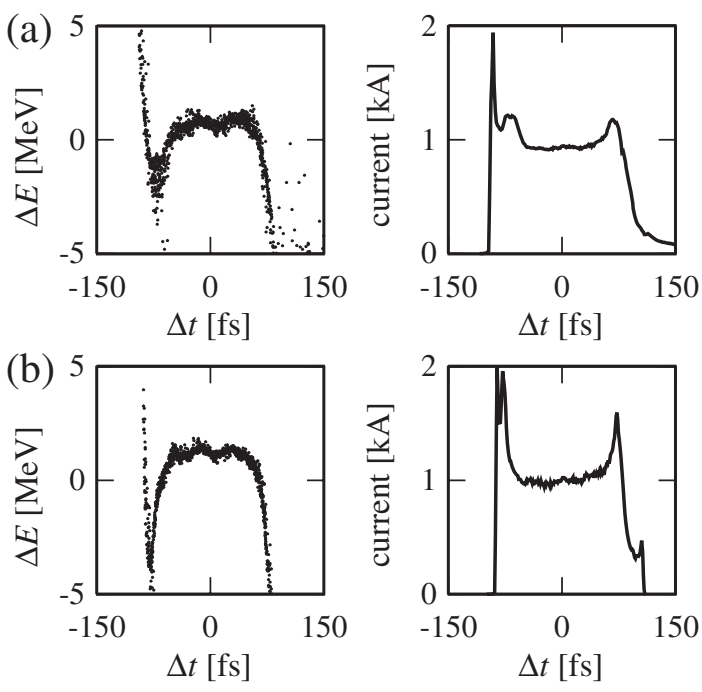

FIG. 9. Simulations of microbunching from shot noise. For oncrest acceleration of the compressed bunch, the left-hand plots show the longitudinal phase space while the right-hand plots show 200-bin current histograms at the exit of the beam spreader. (a) Dechirping by four isochronous arcs at beam energy of $400 \mathrm{MeV}$. (b) Dechirping by four isochronous arcs at beam energy of $1.7 \mathrm{GeV}$. 


\section{SUMMARY}

The energy chirp of a bunch that is undercompressed by a magnetic chicane may be decreased by the CSR wake of several short bending magnets. For a rectangular bunch, we obtained formulas to estimate the amount of dechirping from each bending magnet, and the required distance between the magnets.

For WiFEL, the CSR wake of $\sim 15$ short bending magnets provides the same amount of dechirping as accelerating the compressed bunch $40^{\circ}$ off crest. We developed dechirping schemes in which short magnets are arranged in chicanes or isochronous bending arcs. Tracking simulations show effective dechirping with either arrangement. In simulations of microbunching from shot noise, dechirping with isochronous arcs gives a quieter bunch than dechirping with chicanes.

For WiFEL, dechirping with CSR allows on-crest acceleration of the compressed bunch. For bunches that differ slightly from the design, acceleration slightly before or after crest can provide a dechirped bunch for the FEL.

For FELs in which the required number of bending magnets is not large, dechirping by CSR wakes may be a cost-effective alternative to off-crest acceleration in a linac. In designs where the bunch is compressed at full energy, using CSR wakes to remove the bunch chirp may improve the FEL performance.

\section{ACKNOWLEDGMENTS}

The author thanks K. J. Kleman, K. D. Jacobs, R. A. Legg, M. A. Green, J. J. Bisognano, and J. Wu for helpful discussions. This work is based upon research conducted at the Synchrotron Radiation Center, which is supported by National Science Foundation Award No. DMR-0537588.

[1] M. Cornacchia, S. Di Mitri, G. Penco, and A. A. Zholents, Phys. Rev. ST Accel. Beams 9, 120701 (2006).

[2] J. B. Murphy, S. Krinsky, and R. L. Gluckstern, Part. Accel. 57, 9 (1997).

[3] G. Stupakov and P. Emma, in Proceedings of the Eighth European Particle Accelerator Conference, Paris (EPS-IGA/CERN, Geneva, 2002), p. 1479.

[4] R. A. Bosch, Phys. Rev. ST Accel. Beams 11, 100704 (2008).
[5] M. Venturini and Z. Zholents, Nucl. Instrum. Methods Phys. Res., Sect. A 593, 53 (2008).

[6] J. Qiang, R. D. Ryne, M. Venturini, A. A. Zholents, and I. V. Pogorelov, Phys. Rev. ST Accel. Beams 12, 100702 (2009).

[7] R. Bartolini, C. Christou, J-H. Han, I. P. S. Martin, J. H. Rowland, D. Angal-Kalinin, F. Jackson, B. D. Muratori, and P.H. Williams, in Proceedings of the 2009 Particle Accelerator Conference, Vancouver, Canada (IEEE, Piscataway, NJ, to be published), TU5RFP062.

[8] P. H. Williams, B. D. Muratori, S. L. Smith, J. Rowland, D. Angal-Kalinin, J. K. Jones, H. L. Owen, R. Bartolini, and I. P. Martin, in Proceedings of the 2009 Particle Accelerator Conference, Vancouver, Canada (Ref. [7]), WE5RFP047.

[9] S. V. Benson, D. Douglas, P. Evtushenko, J. Gubeli, F. E. Hannon, K. Jordan, J. M. Klopf, G. R. Neil, M. D. Shinn, C. Tennant, G. P. Williams, and S. Zhang, in Proceedings of the 1st International Particle Accelerator Conference, Kyoto, Japan (IPAC'10/ACFA, Kyoto, Japan, 2010), p. 2302.

[10] P. Craievich, Phys. Rev. ST Accel. Beams 13, 034401 (2010).

[11] R. A. Bosch, J. J. Bisognano, M. Bissen, M. A. Green, H. Höchst, K. D. Jacobs, K. J. Kleman, R. A. Legg, R. Reininger, R. Wehlitz, W.S. Graves, F. X. Kärtner, and D. E. Moncton, in Proceedings of the 31st International Free Electron Laser Conference (FEL 09), Liverpool, UK (STFC Daresbury Laboratory, Warrington, 2009), p. 651.

[12] R. A. Bosch, K. J. Kleman, and J. Wu, in Proceedings of the 2009 Particle Accelerator Conference, Vancouver, Canada (Ref. [7]), WE5RFP058.

[13] M. Borland, Advanced Photon Source Light Source Report No. LS-287, 2000.

[14] J. H. Halton, Numer. Math. 2, 84 (1960).

[15] R. A. Bosch, Phys. Rev. ST Accel. Beams 10, 050701 (2007).

[16] R. A. Bosch, K. J.Kleman, and J. Wu, in Proceedings of the 2009 Particle Accelerator Conference, Vancouver, Canada (Ref. [7]), WE5RFP057.

[17] R. A. Bosch, K. J. Kleman, and J. Wu, in Proceedings of the 31st International Free Electron Laser Conference (FEL 09), Liverpool, UK (Ref. [11]), p. 360.

[18] Z. Huang, M. Borland, P. Emma, J. Wu, C. Limborg, G. Stupakov, and J. Welch, Phys. Rev. ST Accel. Beams 7, 074401 (2004).

[19] E. T. d'Amico and G. Guignard, CERN Report No. CERN/ SL/95-120 (AP), 1995. 